\section{Phrenology revived}

\section{John C. Marshall}

The Modularity of Mind.

By Jerry A. Fodor.

MIT Press/Bradford Books: 1983.

Pp.145. Hbk \$17.50, £15.75;

pbk \$8.50, £7,65.

IN THE popular imagination Franz Joseph Gall (1758-1828) is usually assigned a prominent position in cranks' corner. And indeed a man who could purportedly diagnose talent and character by feeling the bumps on one's skull does not seem too well placed to assume the status of scientific hero.

For the most part, Gall's life and work has supported two industries into whose clutches only a fiend could despatch his worst enemy: first, Gall has been grist to the mill of those philosophers who are convinced that they can infallibly distinguish science from pseudoscience; second, he has provided a happy hunting ground for sociologists who can see parallels between the cultural role that phrenology played in the Victorian era and the recent rise of Californian freak religions. Such are Gall's detractors . . . but his current supporters are typically an even more peculiar lot. The last book I read on phrenology was written by a lady who described herself as "a practising witch".

What then could provoke an eminent Professor of Psychology and Philosophy at MIT to start (albeit metaphorically) "hanging around with phrenologists and other dubious types"? The first thing to make clear is that Jerry Fodor has not taken to the laying on of hands: everyone agrees that craniology was a mistake, although an understandable one in the days before CT scans, positron emission tomography and nuclear magnetic imaging permitted in vivo visualization of the human brain. Rather, Fodor's primary concern is with Gall's views on how the complexity of mental life (and its neuronal substrate) is governed by the operation of a (relatively) small number of discrete components. To appreciate the force and originality of those views we must first look at the tradition Gall came to subvert.

In order to understand the human body we divide it into organs and organ-systems, and into tissues of different types, each with distinct functions. To paraphrase Thomas Hobbes: what is the heart but a pump, and the kidneys but filters; the muscles contractile springs, and the peripheral nerves but so many irritable strings? The basic mechanisms are isolated and the whole interpreted as the interaction of its parts. For at least two millennia, students of cognition have essayed the same strategy: to analyse intellectual capacity as the interplay of diverse primary powers. Methodologically, the paradigm is impeccable. How else could one possibly proceed? But substantively, the utility of the strategy clearly presupposes that we have correctly carved nature "at its joints".

The traditional fractionation of the mind derives from Aristotle and the Early Church Fathers, for whom the faculties of memory, reasoning, attention, perception and abstraction are the fixed inborn components that underly mental activity. These faculties are individuated with respect to their effects, not their subject matter. Thus the principles of memory are,

\section{IMAGE \\ UNAVAILABLE FOR COPYRIGHT REASONS}

on this account, indiscriminately applicable to the storage and recall of events, propositions, faces or melodies; in like fashion, one learns to recognize both roses and Mozart symphonies by abstracting the common elements from the class of exemplars to which one has been exposed; one solves problems in mechanics and the dating of prehistoric artifacts by application of the self-same laws of reasoning to each different data-base.

Contrary to vulgar myth, no exponent of faculty theory ever believed that merely naming the powers of the mind constituted an explanation of those powers; Molière's little joke that opium causes sleep because it possesses "dormative virtue" was singularly misguided. The point of the enterprise was to delimit the activities for which future research might hope to discover the specific nature of the mechanisms responsible for those activities, their quantitative parameters and computational structure. Nor should one imagine that the theory is of merely historical interest. Whenever a modern theoretician postulates a memory system whose capacity is limited to $n$ items, irrespective of what those items are, there lurks a traditional faculty; whenever a perceptual theorist argues that objects in general are recognized by computing the "distance" (in some innate quality space) between the stimulus presented and the members of a set of stored "prototypes", there we have an Aristotelian faculty. In short, Fodor points out, the building blocks of classical psychology and much contemporary psychology are mechanisms whose modus operandi is constant over whatever content is input to them.

And so to Gall. Gall argued, point blank, that the faculties advanced by all previous theoreticians were, in all probability, a fiction. For Gall, there is, as Fodor writes, "no such thing as judgment, no such thing as attention, no such thing as volition, no such thing as memory". A glance at any phrenological bust will confirm that there is literally no overlap with the old faculties. With respect to higher cognitive functions, Gall's innate mental organs are individuated solely in terms of the specific content domain with which they deal. What we find are organs of calculation ( $=$ mental arithmetic), of locality $(=$ the representation and recollection of space and place), of tune (= musical harmony and melody) and of language. Gall did not, of course, deny that one could perceive a melody, attend to a calculation or recall a word. The phrenological credo, repeated in every text, was simply: "That attention, perception, memory, and imagination, are not primitive faculties of the mind, but only modes of activity of all or any of the intellectual faculties". It then follows from Gall's commitment to a punctate locus within the brain for each real faculty that the frontal lobes cannot be the seat of reason, nor the temporal lobes the seat of memory. And hence the fact that organs of tune, locality and language et cetera, are so carefully delineated on phrenological heads. Gall's position also implies that it is entirely an empirical issue whether the mechanisms involved in the perceptual analysis of sentences have anything in common with the mechanisms implicated in the perceptual analysis of faces.

So the fundamental powers that Gall conjectures are domain-specific, genetically determined, associated with discrete neuronal structures and computationally autonomous in that all the machinery necessary for their operation is contained within each distinct mental organ. Fodor coins the term "vertical faculty" to refer to Gall's organs, which leaves him with "horizontal faculty" for 
the traditional concept of domainindependent mechanisms wherein different "contents" compete for limited processing resources. "It seems to me", Fodor writes, "that the notion of a vertical faculty is among the great historical contributions to the development of theoretical psychology", an evaluation with which I fully concur.

A more pointed issue, however, is: are Gall's views correct? Fodor now advances a taxonomy of mentation that specifies those aspects of mind that do seem to be "modular" (in Gall's sense) and those that do not. Fodor distinguishes between transducers (the sense organs that encode proximal stimulation into neural signals), interface systems that compute the structure and character of the distal objects that correspond with particular proximal stimulations, and central systems concerned with the fixation of belief. Interface systems, Fodor argues, are modular, central processes are not. The interface systems are the various mechanisms of domain-specific perceptual analysis plus language, a somewhat "odd category", as Fodor remarks, from the viewpoint of any traditional fractionation of the mind. What does unify the class functionally is that its members all "represent the world as to make it accessible to thought", although they do so in radically distinct ways: "What underwrites the correlation between visual stimulations and distal layouts are (roughly) the laws of light reflectance. Whereas, what underwrites the correlation between token utterances and distal layouts is (roughly) a convention of truth telling". Granted that Fodor's functional characterization is correct, we can then inquire why, in the evolution of the brain, modular systems should have been selected for precisely this class.

The operation of systems that "present the world to thought" must be fast and mandatory if the organism is to survive in a world of predators, moving cars or academic discussion. Interface systems achieve speed by having built in to them a "theory" of the domain to which they are responsive. The algorithm that derives the intrinsic shape of a moving object is constrained by the principle of rigidity: if a collection of moving points has a unique interpretation as a rigid body in motion, "see" that interpretation. Similarly, effective algorithms for parsing sentences must have available to them the structural principles of universal grammar. (Chomsky's Cartesian notion of innately known principles thus fits well with Gall's notion of a "vertical faculty".) The domain-specificity of cognitive modules therefore follows from the specificity of the types of information they must contain.

Fodor further argues that interface systems are "informationally encapsulated" in that their mode of operation does not have access to high-level information about what is likely to be seen or said: "a condition for the reliability of perception, at least for a fallible organism, is that it generally sees what's there, not what it wants or expects to be there. Organisms that don't do so become deceased"'. Fodor's discussion of informational encapsulation draws mainly upon the perception and comprehension of language, where the dominant view (in both artificial intelligence and experimental psychology) is that all kinds of semantic and pragmatic information are brought directly to bear upon the acoustic signal. Fodor's careful unravelling of what exactly the relevant experiments do and don't show should ensure that this free-forall approach is quietly dropped.

In sum, Fodor defends and extends a Gallist approach to the architecture of cognition that is more detailed, more tightly argued and better supported by experimental evidence than Gall himself could have dreamed of. Even at the height of his fame as an anatomist, no respectable scientist would overtly admit the validity of Gall's approach to the fractionation of the mind. Jerry Fodor has never been noticeably respectable, thank goodness; he writes too amusingly for that. But the thesis Fodor advances is the current monograph may well be right. And that, plus having written the most lucid and incisive analysis of theoretical psychology thus far conceived, should be consolation enough.

John C. Marshall is in the Neuropsychology Unit, part of the Neuroscience Group at the Radcliffe Infirmary, Oxford.

\section{Use and abuse of racial differences}

W.F.Bynum

Race and Racism.

By Ruth Benedict.

Routledge \& Kegan Paul: 1983. Pp. 192. $P b k$ £3.95.

OF only one science could it be proposed that its two foremost American practitioners in the middle third of the present century were women. The science is anthropology and the women were Ruth Benedict (1887-1948) and her pupil Margaret Mead (1901-1978). Of the two, Mead undoubtedly had the larger measure of popular success, ultimately becoming a kind of media guru, venerated for her wisdom derived from the study of other societies but called into service in understanding her own. By contrast, Ruth Benedict was of a retiring disposition, and in any case, the fact that she was fourteen years older than her pupil meant that her own academic career was pursued at a time when women rarely occupied chairs in major universities.

Benedict was promoted to a full professorship at Columbia University only shortly before she died. Yet, her book Patterns of Culture (Houghton Mifflin, 1934) has been described as the single most influential work by a twentieth century American anthropologist. It has remained in print and been translated into fourteen languages. In this book, in measured prose and with remarkable insight, she used a series of case studies of American Indian cultures to develop a subtle thesis about the ways in which societies mould the individuals born into them. She pointed out that not just the values and mores, but the perceptions and behavioural strategies can be radically divergent in different cultures: and in her particular case studies, in people who, biologically speaking, belong to the same 'race'.

By the time Benedict published her magnum opus, the Nazis had already come to power, though her book made no reference to the wider world of European politics. Its message, however, was consistent with that of Race and Racism, reprinted now after forty years. Originally published in a somewhat fuller version in America in 1940 as Race: Science and Politics, this book was explicity written for a world drawn into a war in which racism played a central role. Benedict's aims were clear: to explode the myth that race and culture ever have been or ever can be congruent entities, and to argue, using biology, psychology and history, that race can never be used as a valid basis of categorizing individuals.
Prefiguring death - a sketch of Ruth Benedict in 1948 by Erick H. Erikson (from An Anthropologist at Work by Margaret Mead, Houghton Mifflin).

\section{IMAGE \\ UNAVAILABLE FOR COPYRIGHT REASONS}

